\title{
Commentary to: Relative Abundance of Integrant-Derived Viral RNAs in Infected Tissues Harvested from Chronic Hepatitis B Virus Carriers by Freitas N, et al. 2018
}

Stephan Menne ${ }^{1}$ and Severin O. Gudima ${ }^{2^{*}}$

${ }^{1}$ Department of Microbiology and Immunology, Georgetown University Medical Center, Washington, DC, USA

${ }^{2}$ Department of Microbiology, Molecular Genetics and Immunology, University of Kansas Medical Center, Kansas City, Kansas, USA

"Corresponding author: Severin O. Gudima, Associate Professor, Department of Microbiology, Molecular Genetics and Immunology, University of Kansas Medical Center, Room WHW 5031C, 3901 Rainbow Boulevard Kansas City, KS 66160, Tel: (913)-588-6995; E-mail: sgudima@kumc.edu

Received date: Sep 17, 2018; Accepted date: Oct 08, 2018; Published date: Oct 15, 2018

Copyright: (2018 Menne, et al. This is an open-access article distributed under the terms of the Creative Commons Attribution License, which permits unrestricted use, distribution, and reproduction in any medium, provided the original author and source are credited.

\section{Commentary}

During infection with Hepatitis B Virus (HBV), viral DNA is able to integrate into the chromosomal DNA of infected hepatocytes. The main substrate for the integration is double-stranded linear DNA genome of HBV (DSL), which represents approximately $5-20 \%$ of the final products of the reverse transcription catalyzed by the viral polymerase, while the major portion of the final products of HBV reverse transcription is represented by relaxed circular DNA genomes (rcDNA) (Figure 1). The HBV DNA integration is a by-product of viral replication and it is random. Over time, a significant number of infected hepatocytes can acquire HBV DNA integrants [1-3]. Since integrated HBV DNA cannot serve as the source of the progeny virus, it has been commonly assumed that the HBV life cycle is independent of HBV DNA integrants. Using liver and matching hepatocellular carcinoma (HCC) tissues harvested from individuals that were chronically infected with HBV and that were not treated with antiHBV drugs, our recent study demonstrated that RNA transcripts arising from integrated HBV DNA (i.e., HBV integrant-derived RNAs or id-RNAs) were abundantly or even predominantly present, when compared to viral RNAs transcribed from the episomal, covalentlyclosed circular viral DNA (cccDNA) (i.e., replication-derived or rdRNAs) [4]. The observed relative abundance of id-RNAs suggested that these RNA molecules could serve as a considerable source of the HBV envelope proteins (or surface antigen, $\mathrm{HBsAg}$ ) that is independent of the HBV genome replication. Furthermore, our study suggested that id-RNA-derived HBsAg (i.e., id-HBsAg), when abundant as compared to rd-RNA-derived HBsAg (i.e., rd-HBsAg), can regulate the assembly and infectivity of $\mathrm{HBV}$ virions, and thus can influence the ability of HBV to support virus spread and super-infection (Figure 1). The data further suggested that id-RNAs/id-HBsAg therefore can possibly influence the maintenance of the chronic state of HBV infection. In addition, the study proposed to revise the current model of the HBV life cycle by incorporating the potential contributions of id-RNAs and id-HBsAg as described above [4]. Moreover, it became apparent that the inability of current anti-HBV drugs to achieve loss of serum HBsAg in the vast majority of treated patients can be likely explained by significant amounts of id-HBsAg that are translated from id-RNAs independently of HBV replication [4]. Consequently, it is reasonable to suggest that the abundant presence of id-RNAs/id-HBsAg during chronic HBV infection may also have important implications for HBVrelated liver pathogenesis (including carcinogenesis), co- or superinfection with Hepatitis Delta Virus (HDV) [4], and innate and adaptive immunity (Figure 1). The above findings also indicate that the development of new therapeutic interventions directly targeting id-
RNAs and/or id-HBsAg may soon become an important task for the management of chronic HBV infection.

\section{HBV Pathogenesis and Carcinogenesis}

High levels of circulating and intracellular HBsAg can mediate serious liver pathogenesis in HBV-infected patients, including the development of cirrhosis, hepatic decompensation, and HCC [2,5]. Contrary, the risk of developing HCC is significantly reduced in individuals who spontaneously cleared $\mathrm{HBsAg}$ from the circulation (in addition to HBV DNA) or who achieved undetectable serum viremia and antigenemia levels during treatment [6,7]. In regard to these reports, although not demonstrated so far, abundant id-HBsAg, in addition to rd-HBsAg, may substantially account for HBV-associated liver disease progression. Since decades it is known that integrated HBV DNA is present in non-malignant liver tissues as well as in HCC tissues of the same patients [1]. Compared to non-malignant liver tissue from the same patients, a recent report found that HBsAg is differentially expressed in HCC tissues [8]. Although this study did not address the source of the viral protein (i.e., rd- versus id-HBsAg), the expression of the large form of HBsAg (LHBs or the large envelope protein) was unchanged, whereas expression of the small form of the protein (SHBs) was reduced or absent in HCC. Consistent with studies in mouse models of $\mathrm{HBV}[9,10]$, these results indicate that continuously produced wild-type (or mutated) LHBs in the absence of SHBs may accumulate in the endoplasmic reticulum of hepatocytes leading to cellular stress and cytopathic effects thereby promoting HCC development. Mutated LHBs has been indicated in the development of mouse ground glass hepatocytes, which cluster and form nodules and are seen as preneoplastic lesions [9]. Related to this finding, the clonal outgrowth of hepatocytes with limited or undetectable HBV replication, which are not targeted by the immune system, has been implicated in HBV-associated carcinogenesis $[3,11,12]$. Furthermore, since it was shown in cell culture that HBsAg can suppress intracellular HBV genome replication [13], it remains an interesting possibility that at least some hepatocytes involved in this clonal outgrowth may have considerably reduced levels of $\mathrm{HBV}$ replication, because of the production of id-HBsAg. Thus, an immunedriven liver repopulation with initially non-malignant hepatocytes could facilitate HCC onset, when these cells produce id-HBsAg for suppression of HBV replication, and id-HBsAg accumulates overtime within these cells. In addition, it can be speculated that continued production of HBV virions in such hepatocytes, which bear id-HBsAg rather than rd-HBsAg as a major component of their outer envelope, albeit at a very low level, may represent one of the strategies adopted by HBV to maintain chronic infection in the face of immune surveillance, 
Citation: Menne S, Gudima SO (2018) Commentary to: Relative Abundance of Integrant-Derived Viral RNAs in Infected Tissues Harvested from Chronic Hepatitis B Virus Carriers by Freitas N, et al. 2018. Clin Microbiol 7: 318. doi:10.4172/2327-5073.1000318

Page 2 of 5

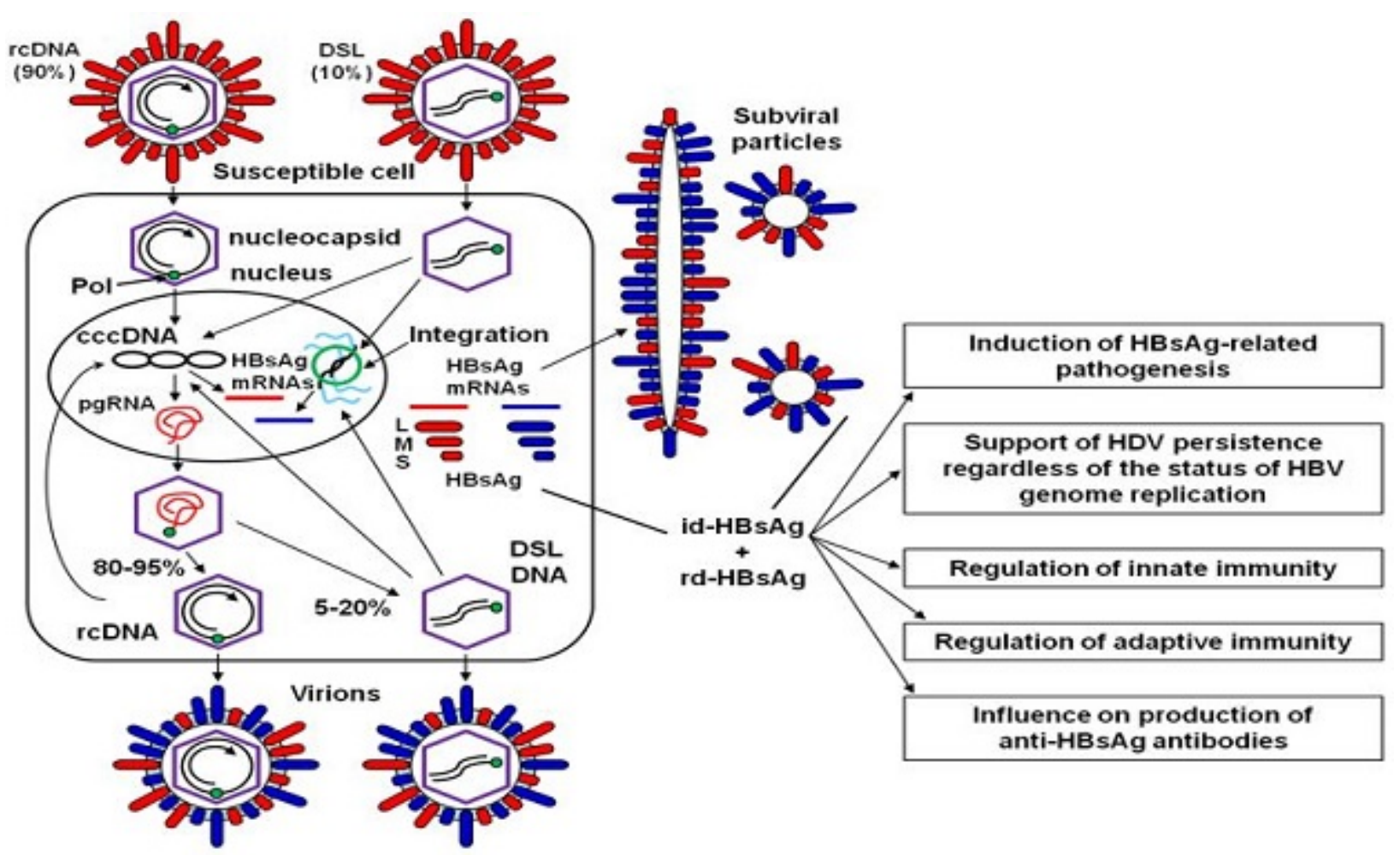

Figure 1: Integrant-derived envelope proteins of HBV (id-HBsAg) and their role in the life cycles of HBV and HDV, HBsAg-related pathogenesis, and interactions with the host immune responses. The presented on the left model of the life cycle of HBV that incorporates production of id-HBsAg and its potential roles and functions is based on the one that was published by Freitas N, et al [4]. About 90\% of HBV virions bear relaxed circular DNA genome (rcDNA) inside the nucleocapsids. The other $\sim 10 \%$ contain double-stranded linear DNA genome (DSL). The HBV entry is a receptor-mediated endocytosis. During the uncoating inside the cell, virion loses the envelope that consists of three viral envelope proteins, large (L), middle $(\mathrm{M})$ and small $(\mathrm{S})$ (shown as bars), and some lipid. Next, DNA synthesis resumes inside the nucleocapsid, and plus DNA strand becomes completed. The polymerase (i.e., reverse transcriptase) of HBV is indicated as a small green circle. After the entry into the nucleus, the nucleocapsid gets disassembled. The rcDNA loses the polymerase, and subsequently gets converted into covalently closed circular DNA (cccDNA) with the help of the host enzymes. The newly-formed cccDNA is the template for synthesis of all RNAs of HBV. One of the RNAs produced by HBV genome replication is pre-genomic RNA (pgRNA). The pgRNA in the complex with viral polymerase gets incorporated into the nucleocapsid made out of HBV core protein molecules. Next, the reverse transcription begins inside the nucleocapsid. The major final product of the reverse transcription ( $~ 80-95 \%$ of cases) is rcDNA. However, in $5-20 \%$ of cases, the final product of the reverse transcription is DSL. Encapsidated rcDNA or DSL can undergo the envelopment with HBsAg and then be released from infected cell as HBV virions. Alternatively, they can be delivered to the nucleus. Inside the nucleus, rcDNA (as mentioned above) is converted into cccDNA. This is how the cccDNA pool gets replenished. Furthermore, DSL is the main substrate for HBV DNA integration. The integration occurs randomly into the host chromosomal DNA and is facilitated by the host DNA repair enzymes [1-4]. Integrated DSL can function as the template for the transcription of HBV integrant-derived RNAs (id-RNAs) encoding HBsAg (independently of HBV genome replication) [4]. The mRNAs for HBsAg are shown as two horizontal lines. The red line represents all HBV replication-derived (i.e., cccDNAderived) mRNAs (rd-RNAs) coding for HBsAg. The blue horizontal line represents all id-RNAs coding for HBsAg. The L, M and S indicate the large, middle and small envelope proteins of HBV, respectively. Shown is the situation, when in infected cell, mRNAs for HBsAg are generated by virus replication and are also produced from integrated HBV DNA. In this case, the envelope proteins are produced from rd-RNAs and from id-RNAs. When id-RNAs are relatively abundant, the newly formed virions of HBV will bear id-RNA-derived HBsAg (id-HBsAg, shown as blue bars) as a major component of their envelopes. Therefore, the properties of these HBV virions will be mainly defined by the functional properties of id-HBsAg, and not by the properties of rd-RNA-derived HBsAg (rd-HBsAg, shown as red bars). Accordingly, id-HBsAg (when abundant) can determine the capability of HBV virions to support virus spread and super-infection that could influence the HBV ability to maintain chronic infection [4]. Also shown are subviral particles that are composed of id-HBsAg and rd-HBsAg, and are released in huge excess as compared to the numbers of HBV virions. Subviral particles come in the forms of small spheres or filaments of various length [2]. Also indicated is the intracellular and secreted HBsAg (of both origins (i.e., id-HBsAg and rd-HBsAg)). On the right part of the figure are important processes that can be influenced by abundant intracellular and/or secreted id-HBsAg (i.e., HBsAg-associated liver pathogenesis, persistence of human Hepatitis Delta Virus (HDV) that uses HBsAg to form its own virions, innate and adaptive immune responses, and production of anti-HBsAg antibodies). 
if the immune system does not efficiently recognize id-HBsAg. Interestingly, one study, which analyzed serum samples from individuals chronically infected with $\mathrm{HBV}$, suggested that circulating antibodies against $\mathrm{HBsAg}$ ( $\mathrm{HBsAb}$ ) did not bind $\mathrm{HBsAg}$, since the HBsAg subtypes present in serum did not display the determinants against which these antibodies were developed, and that this could have prevented the formation of immune complexes [14].

\section{HDV Infection}

Co- and super-infection with HDV contributes substantially to $\mathrm{HBV}$ pathogenesis and carcinogenesis, since this virus mediates liver injury in addition to $\mathrm{HBV}$, induces faster and more frequent development of cirrhosis, and thus increases the overall risk of HCC in $\mathrm{HBV} / \mathrm{HDV}$-infected individuals [15-17]. As a subviral agent of HBV, HDV needs only HBsAg from HBV, but otherwise its life cycle is independent of HBV [17]. Furthermore, persistence of HDV could be independent of HBV replication as previously hypothesized by us [18]. This hypothesis is now further supported by the data from our new study indicating that HBsAg needed for the envelopment of HDV virions may be translated in vivo from id-RNAs (Figure 1) regardless of HBV genome replication [4]. In vitro, we have previously shown that id-HBsAg in the absence of rd-HBsAg supports the production of infectious HDV virions [18]. Use of id-HBsAg rather than rd-HBsAg by HDV could also explain the failure of current anti-HBV drugs to efficiently interfere with HDV infection [19]. Since no drug is available that directly blocks HDV infection [16,19], this underlines the importance of exploring id-RNAs and/or id-HBsAg as targets for the therapeutic intervention of $\mathrm{HDV}$, as well as of $\mathrm{HBV}$, as discussed below.

\section{Innate and Adaptive Immunity}

Several reports suggest that circulating HBsAg may directly regulate innate immunity. HBsAg can suppress plasmacytoid dendritic cell function and affects the transcription of interferon-alpha (IFN- $\alpha$ ) mediated by activation of the toll-like receptor 9 [20]. Internalization of HBsAg by myeloid dendritic cells is associated with a diminished upregulation of costimulatory molecules and reduced $\mathrm{T}$ cell stimulatory capacity [21]. HBsAg has been also shown to suppress the activity of monocytes and to affect cytokine production [22]. Furthermore, the number of natural killer cells declines and cytotoxic cell function becomes attenuated with the expression of HBsAg in the liver of HBV transgenic mice [23]. Circulating HBsAg also affects adaptive immunity since persistent exposure of CD4+ and CD8+ T cells to this protein leads to a stepwise and progressive loss of their effector functions resulting in the overall exhaustion and even deletion of these immune cells [24-26]. Functional impairment of host immunity by HBsAg then in turn renders HBV-infected individuals unable to mount an effective antiviral response that is a prerequisite for HBsAg clearance. The contribution of id-HBsAg in the dysfunction of the above immune cells has not been investigated so far. However, it is known that patients with chronic HBV infection display diminished T cell responses to HBsAg [27], and that reductions in the levels of rdHBsAg by treatment is associated with recovery of this $\mathrm{T}$ cell function [28].

As mentioned above, HBV-infected patients with advanced liver disease and HCC often present with mutations of HBsAg. These commonly found deletions correlate with epitopes affecting CD8+ T cell response and B cell neutralization [29], and thus may help the virus to escape the antiviral immune response. Although not tested as of yet, it is conceivable that id-HBsAg in mutated form, in addition to mutated rd-HBsAg, may contribute to the degree of immune evasion. In this regard, the involvement of humoral response in the control of chronic HBV infection is not very clear. Neutralizing HBsAb are induced in individuals following recovery from acute HBV infection or after immunization with standard vaccines based on recombinant HBsAg, and can inhibit viral attachment and entry [30]. As discussed below, seroconversion to HBsAb is considered a desired endpoint of anti-HBV treatment in individuals with chronic HBV infection. A direct suppression of B cell functions by HBsAg is not known; however, HBV-infected individuals can present with "hyperactive" B cells that show upregulated activation markers and impaired functions [31], and with memory B cells that display reduced proliferative capacity [32]. Thus, (memory) B cell dysfunction may contribute to the immune evasion by HBV.

This overall suggests that high levels of HBsAg can result in the impairment of the host immune response at several distinct levels (Figure 1). Rendering the host unable to mount an effective antiviral response required for clearance of $\mathrm{rd}$ - and id-HBsAg may represent a strategy adopted by HBV to maintain the status of chronic infection in the presence of immune surveillance. Reductions in the level of rdHBsAg have been implicated in the reconstitution of antiviral immune functions in HBV-infected individuals, which has implications for novel interventions against chronic HBV infection.

\section{Development of Therapeutic Interventions for id-RNAs and id-HBsAg}

Current FDA-approved treatment options for chronic HBV infection include five nucleos(t)ide analogs (NUCs) and pegylated IFN- $\alpha$. NUCs are inhibitors of the HBV reverse transcriptase function. They efficiently affect viral genome replication in hepatocytes and can reduce circulating serum HBV DNA to undetectable levels after prolonged treatment. However, the majority of treated patients do not achieve serum HBsAg loss and subsequent seroconversion to $\mathrm{HBs} A \mathrm{~b}$ [33]. Sustained undetectability of viremia and antigenemia (with or without seroconversion) after completion of treatment is a desired endpoint of HBV therapy, since it indicates that the immune system has gained control over the virus leading to a "functional cure" of HBV infection [33]. The reason for the rare HBsAg loss after treatment completion is unclear, but may relate to the narrow mechanism of action of NUCs (i.e., primarily suppression of HBV DNA synthesis) [34]. Another possibility, as discussed above, is that significant HBsAg amounts in patients may be derived from id-RNAs, in addition rdHBsAg (Figure 1). This assumption is supported by a recent study in HBV-infected patients that demonstrated only modest changes in HBsAg serum levels despite undetectable cccDNA in liver following long-term NUC treatment [35]. In line with this finding, the levels of intracellular and circulating id-HBsAg are not expected to markedly change during current antiviral treatment, since NUCs do not directly target id-RNAs. IFN- $\alpha$ also does not directly affect id-RNAs or idHBsAg, but due to its pleiotropic effects on viral and host factors, this cytokine facilitates immune-mediated killing of HBV-infected hepatocytes. Since IFN-mediated removal of HBV replicating hepatocytes also includes those cells with integrated HBV DNA, idHBsAg levels may decline over time, and faster than commonly observed with NUCs. This underlines the need of new therapeutic interventions that directly target id-RNAs for reducing HBsAg, which is independent of rd-RNAs, for treatment of HBV, as well as HDV. Significantly reduced id-HBsAg levels would then contribute to the overall loss of circulating HBsAg thereby allowing restoration of innate 
and adaptive immunity. This could be achieved by treatment with monoclonal antibodies that target specifically id-HBsAg, in case that B cell epitopes are different to those present on rd-HBsAg. Otherwise, antibodies could be used to neutralize HBsAg of both sources from the blood compartment [36]. Expression or secretion of id-HBsAg from hepatocytes may be further inhibited with compounds such as antisense oligonucleotides, small interfering (si)RNA, nucleic acid polymers, and/or synthetic small molecules, which are currently investigated for efficacy against rd-RNA and rd-HBsAg [37-41].

\section{Outcome Expectations for New Therapies Directly Targeting id-RNA and id-HBsAg}

As outlined above, a profound suppression of both sources of HBsAg (i.e., rd-RNAs and id-RNAs) will help achieving the highly desirable outcome of the current guidelines on the management of chronic HBV infection - loss of serum HBsAg [42]. Since loss of HBsAg is considered crucial in the overall restoration of immune functions and in decreasing the risk of associated liver disease progression and HCC, inhibitors of id-RNA/id-HBsAg will most likely need to be combined with conventional NUC treatment or IFN- $\alpha$. Such combinations may induce not only "functional cure", but may be also able to extend the desired treatment outcome into a "complete cure" [42]. In this scenario, rd-and id-HBsAg become undetectable, subsequent seroconversion to $\mathrm{HBsAb}$ is induced, and intrahepatic cccDNA are eradicated following finite treatment duration, all of which would substantially lower the risk of viral reactivation and disease progression.

\section{References}

1. Fowler MJ, Greenfield C, Chu CM, Karayiannis P, Dunk A, et al. (1986) Integration of HBV-DNA may not be a prerequisite for the maintenance of the state of malignant transformation. An analysis of 110 liver biopsies. J Hepatol 2: 218-229.

2. Seeger C, Mason WS (2015) Molecular biology of Hepatitis B virus infection. Virology 479-480: 672-686.

3. Tu T, Mason WS, Clouston AD, Shackel NA, McCaughan GW, et al. (2015) Clonal expansion of hepatocytes with a selective advantage occurs during all stages of chronic Hepatitis B virus infection. J Viral Hepat 22: 737-753.

4. Freitas N, Lukash T, Gunewardena S, Chappell B, Slagle BL, et al. (2018) Relative abundance of integrant-derived viral rnas in infected tissues harvested from chronic Hepatitis B virus carriers. J Virol 92: e02221-17.

5. Suhail M, Abdel-Hafiz H, Ali A, Fatima K, Damanhouri GA, et al. (2014) Potential mechanisms of Hepatitis B virus induced liver injury. World J Gastroenterol 20: 12462-12472.

6. Liu J, Yang HI, Lee MH, Lu SN, Jen CL, et al. (2014) Spontaneous seroclearance of Hepatitis B seromarkers and subsequent risk of hepatocellular carcinoma. Gut 63: 1648-1657.

7. Sung JJ, Tsoi KK, Wong VW, Li KC, Chan HL (2008) Meta-analysis: Treatment of Hepatitis $\mathrm{B}$ infection reduces risk of hepatocellular carcinoma. Aliment Pharmacol Ther 28: 1067-1077.

8. Yen CJ, Ai YL, Tsai HW, Chan SH, Yen CS, et al. Hepatitis B virus surface gene pre-S2 mutant as a high-risk serum marker for hepatoma recurrence after curative hepatic resection. Hepatology 2018.

9. Chisari FV, Klopchin K, Moriyama T, Pasquinelli C, Dunsford HA, et al. (1989) Molecular pathogenesis of hepatocellular carcinoma in Hepatitis B virus transgenic mice. Cell 59: 1145-1156.

10. Teng YC, Neo JC, Wu JC, Chen YF, Kao CH, et al. (2017) Expression of a Hepatitis B virus pre-S2 deletion mutant in the liver results in hepatomegaly and hepatocellular carcinoma in mice. J Pathol 241: 463-474.
11. Mason WS, Liu C, Aldrich CE, Litwin S, Yeh MM (2010) Clonal expansion of normal-appearing human hepatocytes during chronic Hepatitis B virus infection. J Virol 84: 8308-8315.

12. Zoulim F, Mason WS (2012) Reasons to consider earlier treatment of chronic HBV infections. Gut 61: 333-336.

13. Lentz TB, Loeb DD (2011) Roles of the envelope proteins in the amplification of covalently closed circular DNA and completion of synthesis of the plus-strand DNA in Hepatitis B virus. J Virol 85: 11916-11927.

14. Zhang JM, Xu Y, Wang XY, Yin YK, Wu XH, et al. (2007) Coexistence of Hepatitis B surface antigen (HBsAg) and heterologous subtype-specific antibodies to HBsAg among patients with chronic Hepatitis B virus infection. Clin Infect Dis 44: 1161-1169.

15. Fattovich G, Stroffolini T, Zagni I, Donato F (2004) Hepatocellular carcinoma in cirrhosis: incidence and risk factors. Gastroenterology 127: S35-50.

16. Rizzetto M (2015) Hepatitis D Virus: Introduction and Epidemiology. Cold Spring Harb Perspect Med 5: a021576.

17. Taylor JM (2012) Virology of Hepatitis D virus. Semin Liver Dis 32 195-200.

18. Freitas N, Cunha C, Menne S, Gudima SO (2014) Envelope proteins derived from naturally integrated Hepatitis B virus DNA support assembly and release of infectious hepatitis delta virus particles. J Virol 88: 5742-5754.

19. Koytak ES, Yurdaydin C, Glenn JS (2007) Hepatitis D. Curr Treat Options Gastroenterol 10: 456-463.

20. Woltman AM, Op den Brouw ML, Biesta PJ, Shi CC, Janssen HL (2011) Hepatitis B virus lacks immune activating capacity, but actively inhibits plasmacytoid dendritic cell function. PLoS One 6: e15324.

21. Op den Brouw ML, Binda RS, van Roosmalen MH, Protzer U, Janssen HL (2009) Hepatitis B virus surface antigen impairs myeloid dendritic cell function: a possible immune escape mechanism of Hepatitis B virus. Immunology 126: 280-289.

22. Vanlandschoot P, Roobrouck A, Van Houtte F, Leroux-Roels G (2002) Recombinant HBsAg, an apoptotic-like lipoprotein, interferes with the LPS-induced activation of ERK-1/2 and JNK-1/2 in monocytes. Biochem Biophys Res Commun 297: 486-491.

23. Chen Y, Wei H, Sun R, Tian Z (2005) Impaired function of hepatic natural killer cells from murine chronic HBsAg carriers. Int Immunopharmacol 5: 1839-1852.

24. Bertoletti A, Ferrari C (2012) Innate and adaptive immune responses in chronic Hepatitis B virus infections: towards restoration of immune control of viral infection. Gut 61: 1754-1764.

25. Frebel H, Richter K, Oxenius A (2010) How chronic viral infections impact on antigen-specific T-cell responses. Eur J Immunol 40:654-663.

26. Ye B, Liu X, Li X, Kong H, Tian L, et al. (2015) T-cell exhaustion in chronic Hepatitis B infection: current knowledge and clinical significance. Cell Death Dis 6: e1694.

27. Ferrari C (2015) HBV and the immune response. Liver Int 35: 121-128.

28. Boni C, Laccabue D, Lampertico P, Giuberti T, Vigano M, et al. (2012) Restored function of HBV-specific $\mathrm{T}$ cells after long-term effective therapy with nucleos(t)ide analogues. Gastroenterology 143: 963-973 e969.

29. Kao JH, Liu CJ, Jow GM, Chen PJ, Chen DS, et al. (2012) Fine mapping of Hepatitis B virus pre-S deletion and its association with hepatocellular carcinoma. Liver Int 32: 1373-1381.

30. Rehermann B, Nascimbeni M (2005) Immunology of Hepatitis B virus and Hepatitis C virus infection. Nat Rev Immunol 5: 215-229.

31. Xu X, Shang Q, Chen X, Nie W, Zou Z, et al. (2015) Reversal of B-cell hyperactivation and functional impairment is associated with $\mathrm{HBsAg}$ seroconversion in chronic Hepatitis B patients. Cell Mol Immunol 12: 309-316.

32. Oliviero B, Cerino A, Varchetta S, Paudice E, Pai S, et al. (2011) Enhanced $\mathrm{B}$-cell differentiation and reduced proliferative capacity in chronic Hepatitis C and chronic Hepatitis B virus infections. J Hepatol 55: 53-60. 
Citation: Menne S, Gudima SO (2018) Commentary to: Relative Abundance of Integrant-Derived Viral RNAs in Infected Tissues Harvested from Chronic Hepatitis B Virus Carriers by Freitas N, et al. 2018. Clin Microbiol 7: 318. doi:10.4172/2327-5073.1000318

Page 5 of 5

33. Lok AS, Zoulim F, Dusheiko G, Ghany MG (2017) Hepatitis B cure: From discovery to regulatory approval. Hepatology 66: 1296-1313.

34. Werle-Lapostolle B, Bowden S, Locarnini S, Wursthorn K, Petersen J, et al. (2004) Persistence of cccDNA during the natural history of chronic Hepatitis $B$ and decline during adefovir dipivoxil therapy. Gastroenterology 126: 1750-1758.

35. Lai CL, Wong D, Ip P, Kopaniszen M, Seto WK, et al. (2017) Reduction of covalently closed circular DNA with long-term nucleos(t)ide analogue treatment in chronic Hepatitis B. J Hepatol 66: 275-281.

36. Galun E, Eren R, Safadi R, Ashour Y, Terrault N, et al. (2002) Clinical evaluation (phase I) of a combination of two human monoclonal antibodies to HBV: safety and antiviral properties. Hepatology 35: 673-679.

37. Mueller H, Wildum S, Luangsay S, Walther J, Lopez A, et al. (2018) A novel orally available small molecule that inhibits Hepatitis B virus expression. J Hepatol 68: 412-420.

38. Bazinet M, Pantea V, Cebotarescu V, Cojuhari L, Jimbei P, et al. (2017) Safety and efficacy of REP 2139 and pegylated interferon alfa-2a for treatment-naive patients with chronic Hepatitis B virus and Hepatitis D virus co-infection (REP 301 and REP 301-LTF): a non-randomised, openlabel, phase 2 trial. Lancet Gastroenterol Hepatol 2: 877-889.

39. Billioud G, Kruse RL, Carrillo M, Whitten-Bauer C, Gao D, et al. (2016) In vivo reduction of Hepatitis $\mathrm{B}$ virus antigenemia and viremia by antisense oligonucleotides. J Hepatol 64: 781-789.

40. Javanbakht H, Mueller H, Walther J, Zhou X, Lopez A, et al. (2018) Livertargeted anti-HBV single-stranded oligonucleotides with locked nucleic acid potently reduce HBV gene expression in vivo. Mol Ther Nucleic Acids 11: 441-454.

41. Wooddell CI, Yuen MF, Chan HL, Gish RG, Locarnini SA, et al. (2017) RNAi-based treatment of chronically infected patients and chimpanzees reveals that integrated Hepatitis B virus DNA is a source of HBsAg. Sci Transl Med 9: eaan0241.

42. European Association for the Study of the Liver (2017) EASL 2017 Clinical Practice Guidelines on the management of Hepatitis B virus infection. J Hepatol 67: 370-398. 